\title{
LEVANTAMENTO TAXONÔMICO DE ALGAS DE ÁGUA DOCE E CIANOBACTÉRIAS IDENTIFICADOS NA CASCALHEIRA-TRÊS LAGOAS/MS, DURANTE OS PERÍODOS CHUVOSO E SECO.
}

\author{
Mateus Michelan Pieroni ${ }^{1}$ \\ Maria José Neto \\ Rony Carlos Barcelos Blini ${ }^{3}$
}

\begin{abstract}
RESUMO
O local objeto deste trabalho corresponde a uma área que foi utilizada para a remoção de cascalho durante a construção da Usina Hidrelétrica Engenheiro Souza Dias (Jupiá), na década de 1960, conhecida como Cascalheira. O objetivo deste trabalho foi identificar algas e cianobactérias ocorrentes no local durante as estações chuvosa e seca. Para a coleta do material biológico foram realizadas três campanhas durante os meses de julho a dezembro de 2014. As coletas foram feitas em seis pontos com auxílio de uma rede planctônica e em seguida colocadas em recipientes de vidro com tampa e levadas ao laboratório. As amostras foram colocadas entre lâminas e lamínulas e em seguida observadas em um microscópio óptico binocular. A identificação do material deu-se por comparação com literaturas especializadas. Os resultados obtidos permitiram concluir que as algas presentes na cascalheira estão representadas na primeira coleta em 99 espécies distribuídos em 14 classes, na segunda coleta em 119 espécies em 12 classes, e na terceira coleta 141 espécies em 8 classes, e que a quantidade de chuva influência na avaliação quantitativa das mesmas; quanto mais alta a umidade relativa, maior o número de espécies encontradas. A presença dos tipos de classes encontradas demonstra que 0 ambiente passa por processos de eutrofização, mas também indica que o mesmo está em recuperação. Sugere-se a continuidade deste trabalho para futuras comparações, inclusive acrescentando outros parâmetros.
\end{abstract}

\section{RISING WATER ALGAE TAXONOMIC SWEET AND CYANOBACTERIA IDENTIFIED IN GRAVEL - TRÊS LAGOAS / MS , DURING THE PERIODS RAINY AND DRY .}

\footnotetext{
${ }^{1}$ Graduando Ciências Biológicas na Universidade Federal do Mato Grosso do Sul. E-mail: mateuspieroni@hotmail.com

${ }_{2}^{2}$ Doutora em Agronomia da Universidade Federal de Mato Grosso do Sul. maria.neto@ufms.br

${ }^{3}$ Mestre em Geografia pela Universidade Federal de Mato Grosso do Sul
} 


\begin{abstract}
ABTRACT
The local object of this work is an area that was used for the removal of gravel during the construction of the Hydroelectric Plant Engineer Souza Dias (Jupiá), in the 1960s, known as Cascalheira. The objective of this study was to identify algae and cyanobacteria occurring on site during the rainy and dry seasons. For collection of biological material were three campaigns during the months from July to December 2014. The samples were collected at six points with the help of a plankton net and then placed in glass containers with lid and taken to the laboratory. The samples were placed between slides and coverslips and then observed in a binocular optical microscope. The identification of the material was carried out by comparison with specialized literature. The results showed that algae present in the gravel in the first collection are represented by 99 species distributed in 14 classes in the second collection of 119 species in 12 classes, and third collecting 141 species in 8 classes, and that the amount of rain influence the quantitative evaluation; the higher the humidity, the larger the number of species found. The presence of the types of classes found demonstrates that the atmosphere passes through eutrophication processes, but also indicates that it is in recovery. It is suggested to continue this work for future comparisons, including adding other parameters.
\end{abstract}

\title{
LEVANTAMIENTO TAXONÓMICO DE LAS ALGAS DE AGUA DULCE Y CIANOBACTERIAS IDENTIFICADOS EN GRAVA - TRES LAGOAS / MS , DURANTE LOS PERIODOS LLUVIOSO Y SECO.
}

\section{RESÚMEN}

El objeto local de este trabajo es un área que se utilizó para la extracción de grava durante la construcción de la Central Hidroeléctrica Ingeniero Souza Dias (Jupiá), en la década de 1960, conocida como Cascalheira. El objetivo de este estudio fue identificar las algas y cianobacterias que ocurren en el lugar durante las estaciones lluviosas y secas. Para la recolección de material biológico eran tres campañas durante los meses de julio a diciembre de 2014. Se recogieron las muestras en seis puntos con la ayuda de una red de plancton y luego se coloca en recipientes de vidrio con tapa y llevados al laboratorio. Las muestras se colocaron entre las diapositivas y cubreobjetos y luego observadas en un microscopio óptico binocular. La identificación del material se llevó a cabo por comparación con la literatura especializada. Los resultados mostraron que las algas presentes en la grava en la primera colección están representados por 99 especies distribuidas en 14 clases en la segunda colección de 119 especies en 12 clases, y tercero la recogida de 141 especies en 8 clases, y que la cantidad de lluvia influir en la evaluación cuantitativa; cuanto mayor sea la humedad, mayor es el número de especies que se encuentran. La presencia de los tipos de clases se encuentran demuestra que la atmósfera pasa a través de procesos de eutrofización, pero también indica que es en la recuperación. Se sugiere continuar con este trabajo para comparaciones futuras, incluyendo la adición de otros parámetros.

PALABRAS CLAVE: las algas, cascalheira, el fitoplancton 


\section{INTRODUÇÃO}

O termo "alga" foi proposto oficialmente (BICUDO; MENEZES, 2005) como uma categoria taxonômica em 1753, por Lineu, no clássico Species Plantarum. Apresentado no plural como Algae em latim significa "sementes marinhas". Quanto à classificação, um dos sistemas mais conhecidos e aceitos foi proposto por van-DenHoek et al. 1995 e inclui divisões e 30 classes. Baseia-se principalmente na origem endossimbiontica e na ultraestrutura dos cloroplastos, nos tipos de pigmentos fotossintetizantes, na evolução do processo mitótico e na ultraestrutura das raízes flagelares. Nas últimas décadas, novas propostas de sistema de classificação surgiram, englobando dados morfológicos e moleculares. Os caracteres moleculares são interessantes porque mostram uma taxa mais uniforme de evolução do que os caracteres morfológicos e fisiológicos. Enquanto caracteres morfológicos e fisiológicos podem ser influenciados pelas condições ambientais, as sequencias de DNA são entidades hereditárias estáveis, sujeitas unicamente a eventos raros de mutação conforme Johnson et al. 2007. Nos ciclos energéticos dos ecossistemas aquáticos continentais, a comunidade de algas perifíticas desempenha reconhecido papel constituindo um dos principais produtores primários desses ambientes, podendo ainda afetar o crescimento, desenvolvimento, sobrevivência e reprodução de muitos organismos (CAMPEAU et al. 1994).

As alterações na composição taxonômica da comunidade perifítica podem ter um grande significado ecológico, tanto no fluxo de energia, na ciclagem de nutrientes dentre outros processos inerentes aos ecossistemas em planícies de inundação, conforme McCormick et al. 1998.

A composição taxonômica das algas é uma valiosa ferramenta para o conhecimento da integridade biótica e contribui para os diagnósticos das causas diretas e indiretas dos problemas ambientais (STEVENSON; SMOL 2003). Assim, grupos predominantes de algas na comunidade perifítica em determinados ecossistemas podem refletir as características bióticas e abióticas prevalentes no ambiente aquático (PIP; ROBINSON 1981; FELISBERTO et al. 2001). Desta forma, é de fundamental importância o conhecimento taxonômico das algas perifíticas em estudos ecológicos. 
Na década de 1960, fruto da necessidade nacional de geração de energia, edificou-se no município de Três Lagoas MS, a Usina Hidrelétrica Engenheiro Souza Dias (Jupiá). Para esta obra, bem como para vários empreendimentos correlatos e subsequentes, foram extraídos do substrato local, toneladas de matéria-prima mineral. A maior parte das áreas residuais da referida exploração, permanecem como depósitos de rejeitos e escaves profundos sem qualquer tipo de recuperação ambiental. Após o término da construção da usina hidroelétrica, a área utilizada para a remoção de cascalho durante a construção se tornou uma área de segurança, sob os cuidados do Exército e com isso foi utilizada para treinamentos militares. Desde este período, parte da área menos afetada se recuperou e o Cerrado voltou a crescer, tanto que abriga o Parque Municipal das Capivaras. 0 local é um ponto de turismo de Três Lagoas, e a partir de 2004, não mais uma área de segurança.

No local foi escavado um canal para o desvio das águas do rio Sucuriú durante a construção da mesma usina hidroelétrica e devido à proximidade da superfície dos lençóis freáticos da região, os buracos escavados se encheram de água ao longo do tempo, formando lagoas que propiciaram a presença de macrófitas, peixes, organismos aquáticos de modo geral e principalmente algas objeto de estudo deste trabalho.

\section{OBJETIVO}

O objetivo deste trabalho foi identificar algas e cianobactérias ocorrentes no local durante as estações chuvosa e seca.

\section{MATERIAIS E MÉTODOS}

A área de estudo localiza-se na intersecção dos rios Sucuriú e Paraná, na cidade de Três Lagoas-MS. É um complexo formado por várias lagoas denominado Cascalheira, cujas coordenadas geográficas são: Latitude 2045'26.67"S e Longitude $51^{\circ} 39^{\prime} 28.75^{\prime \prime O}$.

Para cada coleta do material biológico foram realizadas campanhas que foram nos períodos: mês de junho com ausência de chuvas, e a temperatura amena, 
em torno de $19^{\circ} \mathrm{C}$, mês novembro com chuvas leves, e a temperatura amena, em torno de $19^{\circ} \mathrm{C}$, e em dezembro já com alta quantidade de chuva alta, com temperaturas amenas, em torno de $19^{\circ} \mathrm{C}$.

A coleta foi feita em seis pontos correspondentes ao sistema UTM, conforme a seguir: PONTO 1 : SETOR: 22k, 04301871 O, $7704500 \mathrm{~N}$, PONTO 2 : SETOR: 22K, 0432034 O, qt0431818 O , 7705373 N, PONTO 5: SETOR: 22K , 0431892 O ,7705177 N, PONTO 6: SETOR: 22K, $043192507704100 \mathrm{~N}$.

Estes pontos foram escolhidos devidos a características exclusivas pertencentes a cada um. Os pontos 1, 2, 3 pertencentes a maior lagoa da Cascalheira, com baixa vegetação, o ponto 4 pertence a uma lagoa do complexo com vegetação densa em sua volta , os pontos 5 ,6 são braços do Rio Paraná. O ponto 5 com pouca vegetação, o ponto 6 pode ser classificado como um braço do rio com circulação praticamente constante de água.

Com auxilio de uma rede planctônica as amostras foram coletadas e em seguida colocadas em recipientes de vidro com tampa e transportadas para o laboratório. As lâminas eram imediatamente preparadas e analisadas ao microscópio óptico binocular Olympus CX40 nos aumentos de 100x e 200x; pelo menos 05 lâminas de cada amostra. As lamínulas foram percorridas transversalmente em toda sua extensão para registrar o maior número possível de táxons.

A preparação, sempre que possível foi analisada com o material ainda fresco de acordo com Bicudo; Menezes (2005), no entanto parte das amostras foi fixada em álcool a 70\% para posteriores averiguações.

A identificação do material, em laboratório, deu-se por comparação com literaturas específicas, entre elas Bicudo; Menezes, 2005, Sant'Anna et al. 2012 e Franceschinni,I.M et al. 2010. 
Figura 1 - Demarcação dos pontos utilizados para coleta-2014.

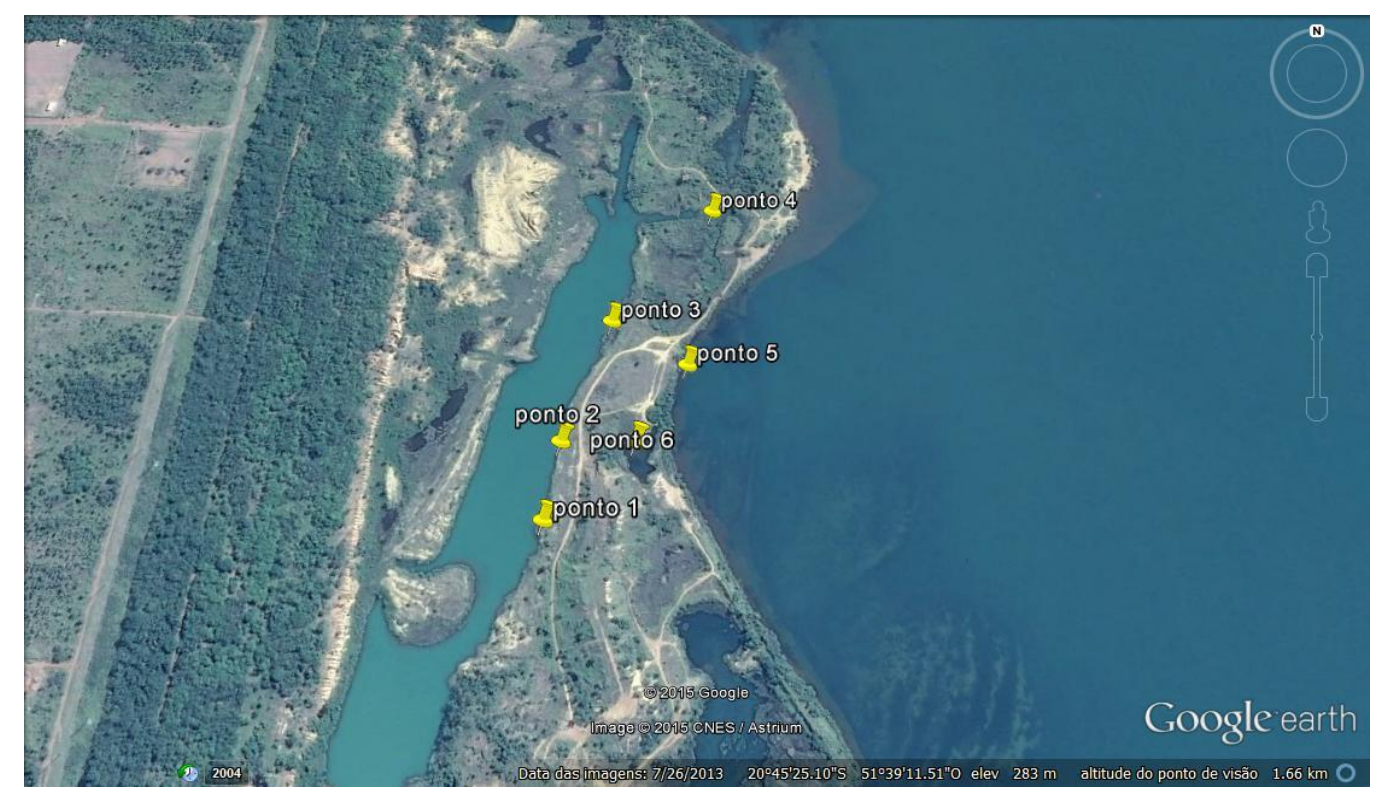

Fonte : Google maps

\section{RESULTADOS E CONCLUSÕES}

Para todas as coletas em seus respectivos pontos, os táxons foram identificados até o nível de espécie e incluídos em suas Classes correspondentes.

Na primeira coleta:

No ponto um, foram identificadas 11 espécies distribuídas em seis grandes grupos denominados taxonomicamente de Classes Desmidiaceae: Hyalotheca dissiliens. Cyanophyceae: Jaaginema lemmermanni. Zygnematophyceae: Mougeotia scalaris, Sphaerozosma aubertianum, Archeri staurastrum, Bambusina brebissonni, Cosmarium caelatum, Staurastrum clavigerum. Conjugatophyceae: Netrium digitus. Diatomophyceae: Surirella angusta. Chlorophyceae: Draparnaldia glomerata,.

No ponto dois, foram identificadas 19 espécies distribuídas em seis Classes Zygnematophyceae: Micrasterias lalicepa, Pleurotaenium tridentulum. Conjugatophyceae: Cylindrocytis brebisonni, Staurastrum rotula, Staurastrum setigerum, Triploceras gracile, Xanthidium regulare, Asteptium borge, Actinoatenium globosum, Cosmarium amoemum, Cosmarium subspeciosum. Cyanophyceae: Pleurotaenium tridentulum, Capitatum stigonema, Tolypotrix tenuis. Chlorophyceae: 
Ulothrix aequalis, Uronema brasiliense, Schizomeris leibleinii.Bacillariophyceae: Craticula halophila, Luticola goeppertiana,.

No ponto três, foram identificadas 16 espécies distribuídas em cinco Classes: Bacillariophyceae: Amphora Montana. Zygnematophyceae: Bambusina brebissonni, Docidium baculum, Desmidium grevillei. Chlorophyceae:, Echinosphaerella limnetica, Tetraedron lobulatum, Asterococus limneticus, Cyanophyceae:, Gymnozyga moniliforms, Synechocystis aquatilis. Conjugatophyceae: Cosmarium boeckii, Cosmarium margaritiferum, Gonatozygon monotaenium, Roya obtusa, Groenbladia neglecta, Micrasterias laticeps.

No ponto quatro, foram identificadas 10 espécies distribuídas em sete Classes: Zygnematophyceae: Bambusina kurzing, Stairastrum gracile, Staurastrum setigerum, Staurastrm setigerum. Trebouxiophyceae: Closteriopsis longíssima. Diatomophyceae: Diadesmis pantropic. Bacillariophyceae: Luticola goeppertiana. Coscinodiscophyceae: Melosira kutizing. Conjugatophycea: Micrasterias laticeps. Cyanophyceae: Rhabdogloea smithii, Microchystis wesenbergii.

No ponto cinco, foram identificadas 18 espécies distribuídas também em sete Classes: Chlorophyceae: Ankistrodesmus bernardii. Zygnematophyceae: Genicularia spirotaenia, Schizomeris leibleinni, Spirogyra maxima, Staurastrum brachioprominens. Conjugatophyceae: Cosmarium subspeciosum, Cosmarium boeckii, Cosmarium boeckii, Euastrum ehrenberg, Haplozyga armata, Staurastrum orbiculare, Staurodesmus Isthmosus, Straurastrum brachioprominens, Micrasterias radians. Charophyceae: Desmidium grevillii. Klebsormidiophyceae: Elakatothrix gelatinosa. Chlorophyceae: Coelastrum polychordum, Desmodesmus maximus, Gymnozyga moniliformis. Coscinodiscophyceae: Melosira nummulodes.

No ponto seis, foram identificadas 25 espécies distribuídas em sete Classes: Chlorophyceae: Ankistrodesmus bernardii, Diacanthos belenophorus, Treubaria triapendiculata. Cyanophyceae: Aphanothece castegnei, Gomphosphaeria aponina, Leptolyngbya lagerheimii, Synechocystis aquatilis. Ulvophyceae: Binuclearia tatrana, Ulothrix aequalis. Bacillariophyceae: Cosmioneis delawarensis. Conjugatophyceae: Micrasterias mahabuleshwarensis, Mougenotia floridana, Shaerozosma aubertianum, Straudesmus glabrus, Actinotaenium globosum, Cylyndrocystis diplospora, Shphaerozosma aubertianumi. Zygnemophyceae: Staurastrum arctisocon, Staurastrum planktinicum, Closterium kuetzingii, Cosmarium corda, 
Shphaerozosma aubertianum. Charophyceae: Desmidium grevillii, Desmidium pseudostreptonema.

Na segunda coleta:

No Ponto um,foram identificadas 25 gêneros em 9 grandes grupos

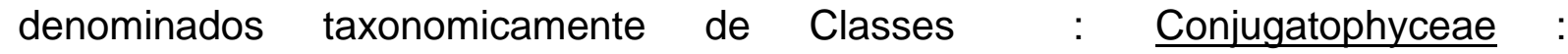
Actinotaenium cucurbitinum. Chlorophyceae: Ankistrodesmus fusiformis,

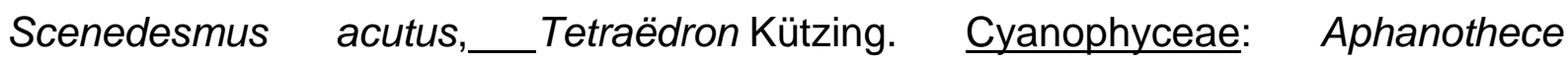
castagnei,__ Leptolyngbya mucicola, Phormidium corium,Pphormidium wilei, Rhabdogloea smithii, Synechococcus aeruginosus. Conjugatophyceae: Bambusina brebissonii, Desmidium pseudostreptonema,_Hyalotheca ehrenberg, Micrasterias radians, Mougeotia floridana, Penium silvae-nigrae, Spirotaenia condensata, Staurastrum claviferum, Staurastrum meyen, Staurastrum orbiculare. Coscinodiscophyceae:___Closteriopsis Lemmermann. Bacillariophyceae: Diadesmis contenta.Fragilariophyceae : Diatoma vulgare. $\underline{\text { Compsopogonophyceae }}$ : Erythrotrichia cárnea.Zygnematophyceae : Pleurotaenium tridentulum.

No Ponto dois,foram identificadas 25 generos em 5 grandes grupos denominados taxonomicamente de Classes: Conjugatophyceae : Actinotaenium cucurbitinum, Actinotaenium globosum, Bambusina kutzing, Closterium moniliferum, Cosmarium granatum, Crucigenia quadrata, Crucigeniella crucífera, Desmidium C.Agardh, Euastrum spinulosum, Spirogyra varians, Staurastrum gracile , Staurodesmus isthmosus. Cyanophyceae : Aphanocapsa pulchra, Chroococcus turgidus,__Coelomoron regulare, Coelomoron micrystoides, Schizomeris leibleinii, Snowella lacustris. Chlorophyceae: Desmodesmus maximus, Desmodesmus protuberans, Gloeocystis ampla, Uronema brasiliense, Volvox aureu. Zygnemophyceae : Micrasterias lalicepa.Ulvophyceae : Ulothrix aequalis.

No Ponto três, foram identificadas 20 generos em 5 grandes grupos denominados taxonomicamente de Classes Conjugatophyceae: Actinotaenium cucurbitum, Actinotaenium globosum, Bambusina armata, Closterium leibleinii, Coelastrum astroideum, Cosmarium reniforme, Cosmarium subtumidum, Docidium baculum, Euastrum pulchellum, Groenbladia neglecta, Mougeotia floridana, Staurastrum setigerum. Cyanophyceae : Chroococcus turgidus, Synechocystis aquatilis. Trebouxiophyceae : Closteriopsis longissima, Tolypothrix tenuis. 
Fragilariophyceae, Diatoma vulgare. Chlorophyceae: Monoraphidium arcuatum, Schroederia setigera, Tetradesmus wisconsinensis.

No Ponto quatro, foram identificadas 21 gêneros em 6 grandes grupos denominados taxonomicamente de Classes Cyanophyceae: Aphanocapsa pulchra, Chroococcus turgidus, Coelosphaerium kuetzingianum, Phormidium puteale, Phormidium willei, Synechococcus aeruginosus.Conjugatophyceae: Bambusina armata, Closterium leibleinii, Cosmarium subtumidum, Netrium digitus, Pleurotaenium cylindricum, Roya obtusa, Spirogyra varians, Spirotaenia condensata, Staurastrum gracile. Chlorophyceae : Chlamydomonas debaryana, Scenedesmus acutus, Schroederia setigera. Trebouxiophyceae: Closteriopsis longíssima. Charophyceae : Nitella translucens .Ulvophyceae : Ulothrix aequalis .

No Ponto cinco, foram identificadas 19 gêneros em 5 grandes grupos denominados taxonomicamente de Classes Conjugatophyceae : Actinotaenium globosum, Actinotaenium cucurbitinum, Bambusina kutzing , Closterium moniliferum, Cosmarium subspeciosum, Micrasterias laticeps, Micrasterias radians, Netrium digitus, Staurodesmus isthmosus, Staurastrum claviferum, Staurastrum setigerum. Trebouxiophyceae : Closteriopsis longissima, Closterium

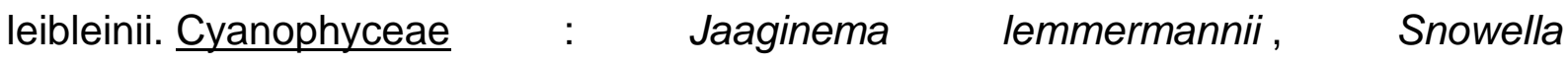
lacustris.Zygnemophyceae : Penium nigrae, Spirogyra columbiana, Spyrogyna varians. Chlorophyceae : Schroederia setigera.

No Ponto seis, foram identificadas 9 gêneros em 6 grandes grupos denominados taxonomicamente de Classes Conjugatophyceae : Desmidium grevillei,Spirotaenia Brébisson. Cyanophyceae: Aphanothece Castegnei, Cylyndrospermopsis Raciborskii, Gloeocapsa Itzigsohnii. Chlorophyceae: Desmodesmus protuberans, Schizomeris leibleinii. Trebouxiophyceae: Closteriopsis Longíssima. Bacillariophyceae: Cosmioneis Delawarensis.

Na terceira coleta:

No Ponto um, foram identificadas 16 gêneros em 5 grandes grupos denominados taxonomicamente de Classes Chlorophyceae : Ankistrodesmus falcatus, Monoraphidium arcuatum, Pandorina morum. Cyanophyceae: Aphanothece castagnei, Planktothrix rubescens, Synechocystis aquatilis . Conjugatophyceae : Bambusina borreri, Cosmarium subtumidum, Groenbladia neglecta, Pleurotaenium tridentulum, Spirogyra varians, Staurastrum 
brachioprominens,

Staurastrum

claviferum,

Staurastrum

setigerum. Fragilariophyceae : Diatoma vulgare.Bacillariophyceae : Rhoicosphenia curvata.

No Ponto dois, foram identificadas 26 gêneros em 5 grandes grupos denominados taxonomicamente de Classes Conjugatophyceae: Actinotaenium cucurbitinum, Bambusina borreri, Closterium leibleinii, Cosmarium granatum, Closterium moniliferum, Cosmarium subtumidum, Desmidium grevillei, Docidium baculum, Groenbladia neglecta, Hyalotheca dissiliens, Micrasterias laticeps, Micrasterias pinnatifida, Micrasterias rotata,_Spirotaenia Brébisson, Spirotaenia Brébisson, Staurastrum claviferum, Staurastrum varians, Staurastrum elegantissimum, Staurastrum setigerum . Cyanophyceae: Aphanocapsa pulchra. .yanophyceae : Aphanothece stagnina, Chroococcus turgidus .Chlorophyceae: Desmodesmus maximus, Monoraphidium arcuatum, Schroederia setigera. Fragilariophyceae : Diatoma vulgare.

No Ponto três, foram identificadas 36 gêneros em 5 grandes grupos denominados taxonomicamente de Classes Conjugatophyceae: Actinotaenium cucurbitinum,_Bambusina Kützing, Actinotaenium globosum, Closterium leibleinii, Closterium longíssima, Closterium moniliferum, Cosmarium subspeciosum, Desmidium grevillei, Docidium baculum, Micrasterias pinnatifida, Micrasterias apiculata, Micrasterias furcata, Netrium digitus, Roya obtusa, Sphaerozosma aubertianum, Staurastrum brebissonii, Staurastrum claviferum, Staurastrum setigeram, Staurodesmus dickiei, Staurodesmus dickiei, Staurodesmus isthmosus, Xanthidium Ehrenberg. Chlorophyceae: Ankistrodesmus densus, Bulbochaete setigera, Eudorina unicocca,_Spirotaenia Brébisson, Tetraëdron minimum, Tetraëdron caudatum. Trebouxiophyceae: Chlorella vulgaris, Closteriopsis longíssima. Cyanophyceae: Chroococcus turgidus, Coelosphaerium, kuetzingianum, Merismopedia convoluta, Synechocystis aquatilis. Zygnematophyceae : spirogyra columbiana.

No Ponto quatro, foram identificadas 36 gêneros em 5 grandes grupos denominados taxonomicamente de Classes: Conjugatophyceae : Actinotaenium cucurbitinum, Closterium leibleinii, _Xanthidium Ehrenberg, Netrium digitus, Staurastrum brachipro, Spirotaenia Brébisson, Staurastrum cuspidatus, Staurastrum brachioprominens, Staurastrum elegantissimus,Staurastrum aeruginosus , 
Xanthidium antilopaeum. Cyanophyceae: Chroococcus limneticus, Eucapsis alpina, Geitlerinema splendidum, Lyngbya mucicola, Lyngbya mucicola, Synechococcus aeruginosus. Trebouxiophyceae: Closteriopsis longissima, closterium moniliferu. Chlorophyceae: Xanthidium Ehrenberg, Schroederia setigera, Tetradesmus wisconsinensis. Fragilariophyceae : Diatoma vulgare.

No Ponto cinco, foram identificadas 18 gêneros em 3 grandes grupos denominados taxonomicamente de Classes: Chlorophyceae : Ankistrodesmus bibraianus, Closterium moniliferum, Microspora palustris, Volvox aureus. Conjugatophyceae : Bambusina borreri, Cosmarium granatum, Cosmarium subspeciosum, Desmidium grevillei, Desmodesmus protuberans, Docidium baculum, Micrasterias laticeps, Spirogyra varians,__Spirotaenia Brébisson, Staurastrum borgensenii, Staurastrum brebissoni.Cyanophyceae : Chroococcus turgidus, Coelosphaerium kuetzingianum, Cylindrospermopsis raciborskii .

No Ponto seis,foram identificadas 11 gêneros em 4 grandes grupos denominados taxonomicamente de Classes: Conjugatophyceae : Desmidium grevillei ,Spirotaenia Brébisson, Pleurotaenium ehrenbergii , Staurastrum elegantissimum, Staurastrum setigerum. Cyanophyceae: Jaaginema lemmermannii, Rhabdogloea smithii.Chlorophyceae: Desmodesmus protuberans, Schizomeris leibleinii.Zygnematophyceae : Spirogyra columbiana, Spirogyra varians. 
Quadro 1 - Quantidade total de algas por coleta

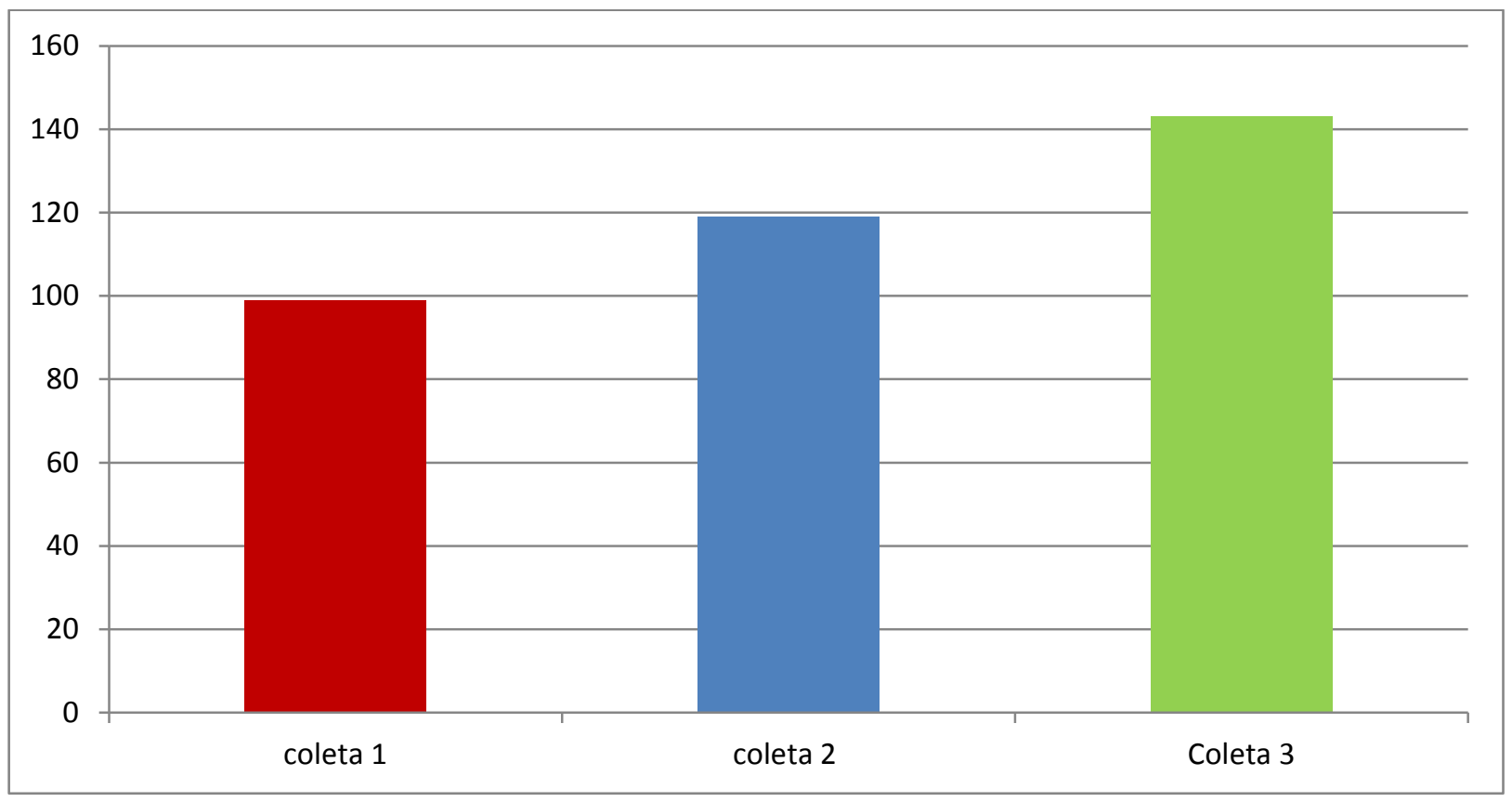

Quadro 2 - Quantidade de classes para cada ponto por coleta

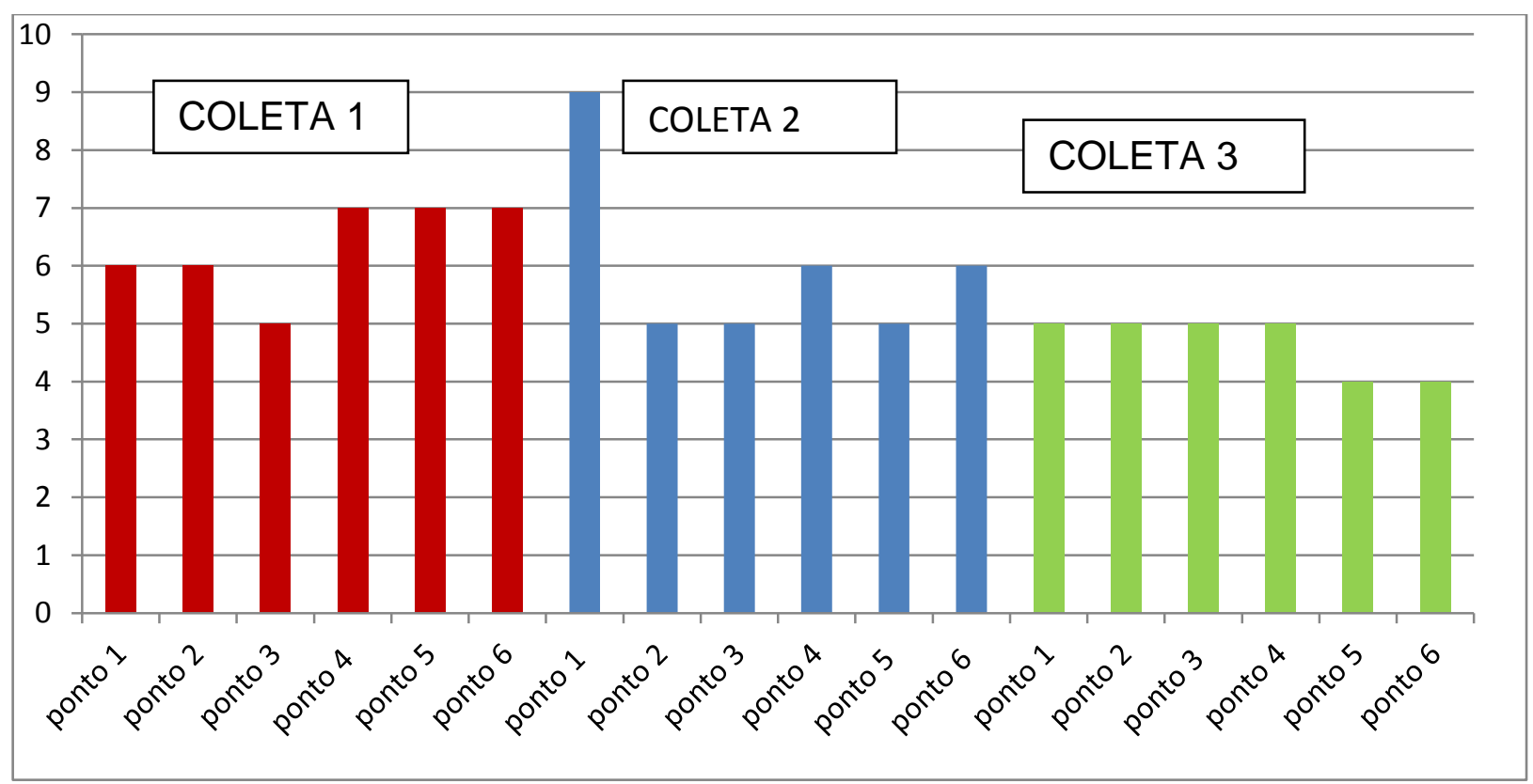

\section{CONSIDERAÇÕES FINAIS}

Os dados obtidos permitiram concluir que a quantidade de chuva influencia na avaliação quantitativa das algas e cianobactérias, sendo que quanto mais úmido o 
ambiente, será maior a riqueza encontrada (Quadro 1 - quantidade total de algas por coleta). Durante as observações notou-se que apesar de terem sido realizadas três campanhas em estações distintas e pontuais, não houve variações dos gêneros encontrados, e quanto ao numero de classes esse dentro da campanha ficaram constantes, mas entre uma campanha e outra houve uma pequena variação (Quadro 2 - Quantidade de classes para cada ponto por coleta). Já composição do fitoplâncton das lagoas do complexo Cascalheira é relativamente similar aos lagos de inundação de outras áreas do Brasil, de acordo com (HENRY et al, 2006), normalmente constituída pelos grandes grupos taxonômicos constituídos predominantemente pelas classes Euglenophyceae, Chlorophyceae, como no Lago Castelo e no Rio Paraguai (OLIVEIRA; CALHEIROS, 2000), por Chlorophyceae e Bacillariophyceae como no Rio Baía no Alto Paraná (TRAIN; RODRIGUES, 1998), e por Zygnemaphyceae e Chlorophyceae na Lagoa Albuquerque no Pantanal conforme (ESPINDOLA et al. 1996). Numericamente as classes mais representativas coincidem com os trabalhos citados, as demais classes encontradas com baixo percentual indicam ser particularidades da área em questão. $E$ ainda deve se considerar a presença de Zygnematophyceae em alto nível nos pontos 1, 2 e 3 ,em todos as coletas o que indica que o ambiente que esta se restituindo , já sofre de eutrofização, o que esta de acordo com toda literatura sobre corpos hídricos eutrofizados de regiões tropicais

A presença destes organismos pode indicar que o ambiente está em recuperação, mas sugere-se a continuidade deste trabalho para futuras comparações com outros parâmetros, principalmente com a análise físico-química da água. 


\section{REFERÊNCIAS}

BICUDO, C.E.; MENEZES, M. Gêneros de algas de águas continentais do Brasil: chave para identificação e descrições. Editora Rima. 2005.

CAMPEAU, S.; MURKIN, H.R. ; TITMAN, R.D. 1994. Relative importance of algae and emergent plant litter to freshwater marsh invertebrates. Canadian Journal Fisheries and Aquatic Sciences, 51:681692.

ESPÍNDOLA, E.G.; MATSUMURA-TUNDISI, T.; MORENO, I.D. 1996. Estrutura da comunidade fitoplanctônica da lagoa Albuquerque (Pantanal Mato-grossense), Mato Grosso do Sul, Brasil. Acta Limnologica Brasiliensia, 8: 13-27.

FELISBERTO, S.A.; RODRIGUES, L.; LEANDRINI, J.A. 2001. Chloroccoccales registradas na comunidade perifitica no reservatório Corumbá, Estado de Goiás, Brasil, antes e após o represamento das águas. Acta Scientiarium, 23:275-282.

FRANCESCHINNI, I.M; BURLIGA, A.I.: Reviers, B.; PRADO J.F \& HAMLAQUI; S.2009. Algas - Uma abordagem filogenética, taxonômica e ecológica. Porto Alegre: Artmed.

HENRY, R.; USHINOHAMA, E.; FERREIRA, R.M.R.. Fitoplâncton em três lagoas marginais ao Rio Paranapanema e em sua desembocadura no Reservatório de Jurumirim (São Paulo, Brasil) durante um período prolongado de seca. Revista Brasil. Bot, V.29, n.3, p.399-414, jul-set 2006.

JOHNSON, D.S.; MORTAZAVI, A.; MYERS R.M. and WOLD,B., et al. (2007).Genome-wide mapping of in vivo protein-DNA interactions. Science,316, 1497-1502

LINNAEUS, C. 1753. Species Plantarum 1ำed., v.2, p.1009.The Ray Society London (Facsimile 1959).

OLIVEIRA, M.D.; CALHEIROS, D.F. 2000. Flood pulse influence in phytoplankton communities of the south Pantanal floodplain, Brazil. Hydrobiologia 427:101-112.

PIP, E. ; ROBINSON, G.G.C. 1981. A comparision of algal periphyton composition on eleven species of submerged macrophytes. Hydrobiological Bulletin 18:109-118.

Sant'Anna, C.L.; Tucci, A.; Azevedo, M.T.P.; Melcher, S.S.; Werner, V.R.; Malone, C.F.S.; Rossini, E.F.; Jacinavicius, F.R.; Hentschke, G.S.; Osti, J.A.S.; Santos, K.R.S.; Gama-Júnior, W.A.; Rosal, C. \& Adame, G. 2012. Atlas de cianobactérias e microalgas de águas continentais brasileiras. Publicação eletrônica, Instituto de Botânica, Núcleo de Pesquisa em Ficologia. www.ibot.sp.gov.br 
STEVENSON , R.J. ; SMOL, J.P. 2003. Use of algae in environmental assessments. In Freshwater algae of North America, ecology and classification (J.D. Wehr \& Sheath, R.G., eds.). Academic Press, San Diego, p.775-804.

TRAIN, S., Oliveira, M.D. ; Quevedo, M.T. 2000. Dinâmica sazonal da comunidade fitoplanctônica de um canal lateral (Canal Cortado) do Alto Rio Paraná. Acta Scientiarum 22:389-399.

VAN DEN HOEK, C; MANN, D.G ; JANHNS, H.M. 1995. Algae: an introduction to phycology. Cambridge, Cambridge University Press. 